\title{
Crystal Structure of 2-Dibenzoylmethyl Benzimidazole
}

\author{
Sema Öztürk, ${ }^{* \dagger}$ Mehmet AKKurt,* Mehmet Sönmez,** and Semra İDE*** \\ *Department of Physics, Faculty of Arts and Sciences, Erciyes University, Kayseri-38039, Turkey \\ **Department of Chemistry, Faculty of Arts and Sciences, Yüzüncü Yll University, Van-65080, Turkey \\ ***Department of Physics Engineering, Faculty of Engineering, Hacettepe University, \\ Beytepe, Ankara-06532, Turkey
}

(Received September 22, 2000; Accepted June 15, 2001)

\begin{abstract}
Many substituted benzimidazoles are currently used in the treatment of various infections caused by fungi, bacteria, nematodes and viruses. ${ }^{1}$ The benzimidazole ring is included in biologically active substances and in a variety of clinically useful drugs such as Omeprazole, Astemizole, Emedastine difumarate, and Cyanocobalamine. ${ }^{2}$ In particular, during the last decade, the antiallergic and antihistaminic activity of the benzimidazoles has received much attention. ${ }^{3}$

The title compound was prepared by the reaction of dibenzoylacetic acid- $N$-carboxymethylamide and $O$ phenylenediamine. Measured amounts $(0.650 \mathrm{~g}$ of dibenzoylacetic acid- $N$-carboxymethylamide and $0.220 \mathrm{~g}$ of $o$ phenylenediamine) were joined in $30 \mathrm{ml}$ of xylene. This
\end{abstract}

Table 1 Crystal and experimental data

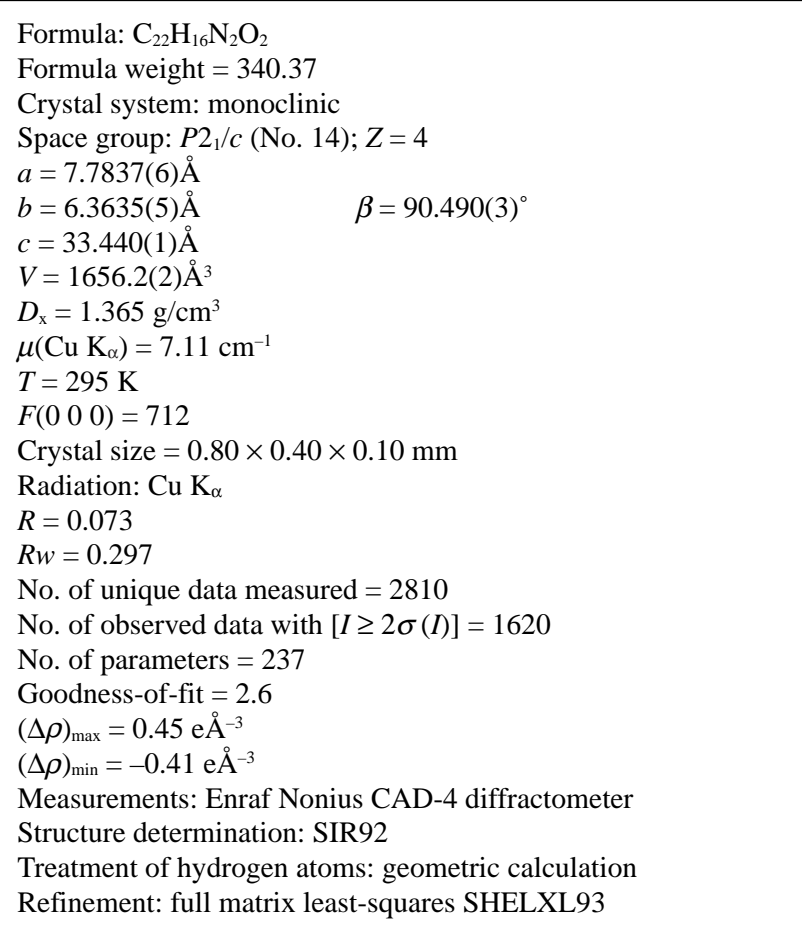

$\uparrow$ To whom correspondence should be addressed.

E-mail: ozturk@erciyes.edu.tr mixture was heated in a reflux for $4 \mathrm{~h}$. After the solvent was removed from the rotovapor, the precipitate so formed was recrystallized from absolute ethanol (Fig. 1).

The product shows a keto-enol tautomery. In this study, we describe the single crystal structure analysis of the title compound with a keto-form in solid phase. A summary of the key crystallographic information is given in Table 1. The atomic coordinates and equivalent isotropic displacement parameters for non-hydrogen atoms are listed in Table 2, and

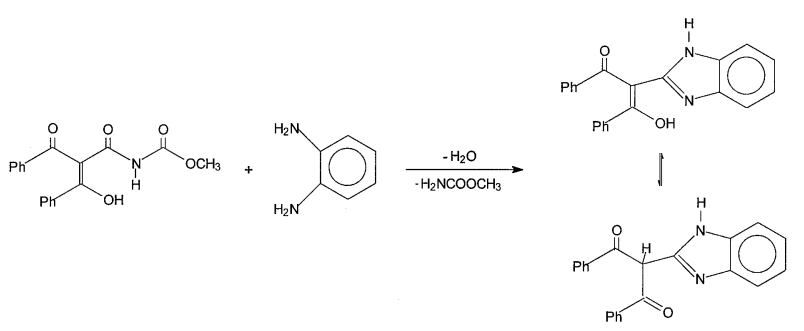

Fig. 1 Synthesis pathway of the title compound.

Table 2 Final atomic coordinates and equivalent isotropic thermal displacement parameters for non-hydrogen atoms

\begin{tabular}{|c|c|c|c|c|}
\hline Atom & $x$ & $y$ & $z$ & $U_{\text {eq }}$ \\
\hline 09 & $0.408(2)$ & $-0.021(2)$ & $0.4213(3)$ & $0.082(4)$ \\
\hline 010 & $0.475(2)$ & $0.599(2)$ & $0.3479(3)$ & $0.088(4)$ \\
\hline N1 & $0.584(2)$ & $0.280(2)$ & $0.4527(4)$ & $0.077(4)$ \\
\hline N2 & $0.608(2)$ & $0.576(2)$ & $0.4195(3)$ & $0.067(3)$ \\
\hline $\mathrm{C} 1$ & $0.677(2)$ & $0.418(2)$ & $0.4777(4)$ & $0.069(4)$ \\
\hline $\mathrm{C} 2$ & $0.743(2)$ & $0.395(3)$ & $0.5141(4)$ & $0.072(4)$ \\
\hline C3 & $0.830(2)$ & $0.565(3)$ & $0.5324(5)$ & $0.074(4)$ \\
\hline $\mathrm{C} 4$ & $0.849(2)$ & $0.752(3)$ & $0.5110(5)$ & $0.081(5)$ \\
\hline $\mathrm{C} 5$ & $0.780(2)$ & $0.772(2)$ & $0.4723(4)$ & $0.069(4)$ \\
\hline C6 & $0.696(2)$ & $0.604(2)$ & $0.4555(4)$ & $0.057(4)$ \\
\hline $\mathrm{C} 7$ & $0.541(2)$ & $0.383(2)$ & $0.4178(4)$ & $0.064(4)$ \\
\hline $\mathrm{C} 8$ & $0.434(2)$ & $0.434(2)$ & $0.3876(4)$ & $0.058(4)$ \\
\hline C9 & $0.350(2)$ & $0.350(2)$ & $0.3958(4)$ & $0.064(4)$ \\
\hline C90 & $0.168(2)$ & $0.168(2)$ & $0.3793(4)$ & $0.067(4)$ \\
\hline C91 & $0.048(2)$ & $0.048(2)$ & $0.3692(4)$ & $0.069(4)$ \\
\hline C92 & $-0.113(2)$ & $-0.113(2)$ & $0.3594(4)$ & $0.068(4)$ \\
\hline C93 & $-0.167(2)$ & $-0.167(2)$ & $0.3572(4)$ & $0.082(5)$ \\
\hline C94 & $-0.046(2)$ & $-0.046(2)$ & $0.3674(5)$ & $0.081(5)$ \\
\hline C95 & $0.111(2)$ & $0.111(2)$ & $0.3775(4)$ & $0.067(4)$ \\
\hline C10 & $0.422(2)$ & $0.422(2)$ & $0.3490(4)$ & $0.062(4)$ \\
\hline $\mathrm{C} 100$ & $0.352(2)$ & $0.352(2)$ & $0.3136(4)$ & $0.069(4)$ \\
\hline $\mathrm{C} 101$ & $0.253(2)$ & $0.253(2)$ & $0.2875(5)$ & $0.087(5)$ \\
\hline C102 & $0.192(3)$ & $0.192(3)$ & $0.2506(5)$ & $0.096(6)$ \\
\hline C103 & $0.230(3)$ & $0.230(3)$ & $0.2401(6)$ & $0.108(7)$ \\
\hline $\mathrm{C} 104$ & $0.332(3)$ & $0.332(3)$ & $0.2647(5)$ & $0.089(5)$ \\
\hline C105 & $0.391(2)$ & $0.391(2)$ & $0.3017(5)$ & $0.073(4)$ \\
\hline
\end{tabular}

$\overline{U_{\text {eq }}}=(1 / 3) \sum_{i} \Sigma_{j} U_{i j} a_{i} * a_{j}^{*}\left(\boldsymbol{a}_{i} \cdot \boldsymbol{a}_{j}\right)$. 
Table 3 Bond lengths $(\AA)$, bond and torsion angles $\left({ }^{\circ}\right.$ )

\begin{tabular}{|c|c|c|c|}
\hline $\mathrm{O} 9-\mathrm{C} 9$ & $1.24(2)$ & $\mathrm{C} 9-\mathrm{C} 90$ & $1.55(2)$ \\
\hline $\mathrm{O} 10-\mathrm{C} 10$ & $1.21(2)$ & $\mathrm{C} 90-\mathrm{C} 95$ & $1.40(2)$ \\
\hline $\mathrm{N} 1-\mathrm{C} 7$ & $1.37(2)$ & $\mathrm{C} 90-\mathrm{C} 91$ & $1.41(2)$ \\
\hline $\mathrm{N} 1-\mathrm{C} 1$ & $1.41(2)$ & $\mathrm{C} 91-\mathrm{C} 92$ & $1.33(2)$ \\
\hline $\mathrm{N} 2-\mathrm{C} 7$ & $1.34(2)$ & $\mathrm{C} 92-\mathrm{C} 93$ & $1.37(2)$ \\
\hline $\mathrm{N} 2-\mathrm{C} 6$ & $1.39(2)$ & $\mathrm{C} 93-\mathrm{C} 94$ & $1.40(2)$ \\
\hline $\mathrm{C} 1-\mathrm{C} 2$ & $1.32(2)$ & $\mathrm{C} 94-\mathrm{C} 95$ & $1.29(2)$ \\
\hline $\mathrm{C} 1-\mathrm{C} 6$ & $1.41(2)$ & $\mathrm{C} 10-\mathrm{C} 100$ & $1.41(2)$ \\
\hline $\mathrm{C} 2-\mathrm{C} 3$ & $1.41(2)$ & $\mathrm{C} 100-\mathrm{C} 101$ & $1.36(2)$ \\
\hline $\mathrm{C} 3-\mathrm{C} 4$ & $1.40(2)$ & $\mathrm{C} 100-\mathrm{C} 105$ & $1.49(2)$ \\
\hline $\mathrm{C} 4-\mathrm{C} 5$ & $1.40(2)$ & $\mathrm{C} 101-\mathrm{C} 102$ & $1.39(2)$ \\
\hline $\mathrm{C} 5-\mathrm{C} 6$ & $1.37(2)$ & $\mathrm{C} 102-\mathrm{C} 103$ & $1.36(3)$ \\
\hline $\mathrm{C} 7-\mathrm{C} 8$ & $1.42(2)$ & $\mathrm{C} 103-\mathrm{C} 104$ & $1.38(2)$ \\
\hline $\mathrm{C} 8-\mathrm{C} 9$ & $1.42(2)$ & $\mathrm{C} 104-\mathrm{C} 105$ & $1.38(2)$ \\
\hline $\mathrm{C} 8-\mathrm{C} 10$ & $1.52(2)$ & & \\
\hline $\mathrm{C} 7-\mathrm{N} 1-\mathrm{C} 1$ & $109(1)$ & $\mathrm{C} 8-\mathrm{C} 9-\mathrm{C} 90$ & $121(1)$ \\
\hline $\mathrm{C} 7-\mathrm{N} 2-\mathrm{C} 6$ & $110(1)$ & $\mathrm{C} 95-\mathrm{C} 90-\mathrm{C} 91$ & $118(2)$ \\
\hline $\mathrm{C} 2-\mathrm{C} 1-\mathrm{N} 1$ & $132(2)$ & $\mathrm{C} 95-\mathrm{C} 90-\mathrm{C} 9$ & $118(1)$ \\
\hline $\mathrm{C} 2-\mathrm{C} 1-\mathrm{C} 6$ & $122(1)$ & $\mathrm{C} 91-\mathrm{C} 90-\mathrm{C} 9$ & $124(1)$ \\
\hline $\mathrm{N} 1-\mathrm{C} 1-\mathrm{C} 6$ & $105(1)$ & $\mathrm{C} 92-\mathrm{C} 91-\mathrm{C} 90$ & $121(2)$ \\
\hline $\mathrm{C} 1-\mathrm{C} 2-\mathrm{C} 3$ & $120(1)$ & $\mathrm{C} 91-\mathrm{C} 92-\mathrm{C} 93$ & $122(2)$ \\
\hline $\mathrm{C} 4-\mathrm{C} 3-\mathrm{C} 2$ & $119(1)$ & $\mathrm{C} 92-\mathrm{C} 93-\mathrm{C} 94$ & $116(2)$ \\
\hline $\mathrm{C} 3-\mathrm{C} 4-\mathrm{C} 5$ & $121(2)$ & $\mathrm{C} 95-\mathrm{C} 94-\mathrm{C} 93$ & $124(2)$ \\
\hline $\mathrm{C} 6-\mathrm{C} 5-\mathrm{C} 4$ & $119(1)$ & $\mathrm{C} 94-\mathrm{C} 95-\mathrm{C} 90$ & $119(2)$ \\
\hline $\mathrm{C} 5-\mathrm{C} 6-\mathrm{N} 2$ & $133(1)$ & $\mathrm{O} 10-\mathrm{C} 10-\mathrm{C} 100$ & $118(1)$ \\
\hline $\mathrm{C} 5-\mathrm{C} 6-\mathrm{C} 1$ & $119(1)$ & $010-\mathrm{C} 10-\mathrm{C} 8$ & $120(1)$ \\
\hline $\mathrm{N} 2-\mathrm{C} 6-\mathrm{C} 1$ & $107(1)$ & $\mathrm{C} 100-\mathrm{C} 10-\mathrm{C} 8$ & $122(1)$ \\
\hline $\mathrm{N} 2-\mathrm{C} 7-\mathrm{N} 1$ & $108(1)$ & $\mathrm{C} 101-\mathrm{C} 100-\mathrm{C} 10$ & $123(2)$ \\
\hline $\mathrm{N} 2-\mathrm{C} 7-\mathrm{C} 8$ & $128(1)$ & $\mathrm{C} 101-\mathrm{C} 100-\mathrm{C} 105$ & $116(2)$ \\
\hline $\mathrm{N} 1-\mathrm{C} 7-\mathrm{C} 8$ & $124(1)$ & $\mathrm{C} 10-\mathrm{C} 100-\mathrm{C} 105$ & $121(1)$ \\
\hline $\mathrm{C} 7-\mathrm{C} 8-\mathrm{C} 9$ & $118(1)$ & $\mathrm{C} 100-\mathrm{C} 101-\mathrm{C} 102$ & $125(2)$ \\
\hline $\mathrm{C} 7-\mathrm{C} 8-\mathrm{C} 10$ & $116(1)$ & $\mathrm{C} 103-\mathrm{C} 102-\mathrm{C} 101$ & $118(2)$ \\
\hline $\mathrm{C} 9-\mathrm{C} 8-\mathrm{C} 10$ & $126(1)$ & $\mathrm{C} 102-\mathrm{C}_{103}-\mathrm{C} 104$ & $120(2)$ \\
\hline $\mathrm{O} 9-\mathrm{C} 9-\mathrm{C} 8$ & $121(1)$ & $\mathrm{C} 105-\mathrm{C} 104-\mathrm{C} 103$ & $124(2)$ \\
\hline $\mathrm{O} 9-\mathrm{C} 9-\mathrm{C} 90$ & $117(1)$ & $\mathrm{C} 104-\mathrm{C} 105-\mathrm{C} 100$ & $117(1)$ \\
\hline
\end{tabular}

bond lengths and bond angles are given in Table 3. Figure 2 represents the molecular structure of the title compound.

In the title compound, $\mathrm{N} 1-\mathrm{C} 7$ and $\mathrm{N} 2-\mathrm{C} 7$ bond lengths are

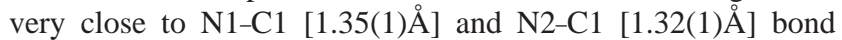
lengths in the bis(methyl-3-ethyl-benzimidazolidine-2-ylium) tetrafluoroborate. ${ }^{4} \mathrm{~N} 1-\mathrm{C} 7$ bond length $[1.37(2) \AA]$ is longer than N2-C7 bond length [1.34(2) $\mathrm{A}]$, the corresponding values, in 1ethyl-3-methylbenzimidazole-2-thione, ${ }^{5} \quad 1.364(5)$ and 1.349(5) $\mathrm{A}$, in 2-(3-methoxy-2-hydroxyphenyl)benzimidazole, ${ }^{6}$ are 1.371(4) and 1.325(5) $\AA$; and in 1-(phenylmethyl)-2-(4methoxyphenylmethyl)- $1 H$-benzimidazole-5-carboxylic acid, ${ }^{7}$ 1.365(4) and 1.331(4) $\AA$, respectively. The benzimidazole ring system is almost planar, with a dihedral angle of $2.1(5)^{\circ}$ between the imidazole and benzene ring planes. The angles $\mathrm{N} 2-\mathrm{C} 7-\mathrm{C} 8=128(1)$ and $\mathrm{N} 1-\mathrm{C} 7-\mathrm{C} 8=124(1)^{\circ}$ are almost symmetric.

The structure is stabilized by one intra and two intermolecular hydrogen bonds, which are given in Table 4. For a structure analysis, $U_{\text {eq }}$ normally has values about $0.05 \AA^{2}$ at room temperature. The high $U_{\text {eq }}$ values for some atoms in Table 2 may indicate a low quality of the investigated crystal.
Table 4 Possible hydrogen bond lengths $(\AA)$ and angles ( $\left.{ }^{\circ}\right)$

\begin{tabular}{|c|c|c|c|c|}
\hline $\mathrm{D}-\mathrm{H} \cdots \mathrm{A}$ & D-H $(\AA)$ & $\mathrm{H} \cdots \mathrm{A}(\AA)$ & $\mathrm{D} \cdots \mathrm{A}(\AA)$ & $\mathrm{D}-\mathrm{H} \cdots \mathrm{A}\left({ }^{\circ}\right)$ \\
\hline $\mathrm{H} 2-\mathrm{H} 2 \mathrm{~N} \ldots O 9^{i}$ & $0.86(2)$ & $2.56(2)$ & $3.00(2)$ & $113(1)$ \\
\hline $\mathrm{N} 2-\mathrm{H} 2 \mathrm{~N} \ldots \mathrm{O} 10$ & $0.86(2)$ & $2.07(2)$ & $2.61(2)$ & $120(1)$ \\
\hline $\mathrm{C} 2-\mathrm{H} 2 \ldots O 9^{i \mathrm{i}}$ & $0.93(3)$ & $2.57(2)$ & $3.43(2)$ & $154(2)$ \\
\hline
\end{tabular}

Equivalent positions: $[i=x, 1+y, z ; i i=1-x, 1-y,-z]$.

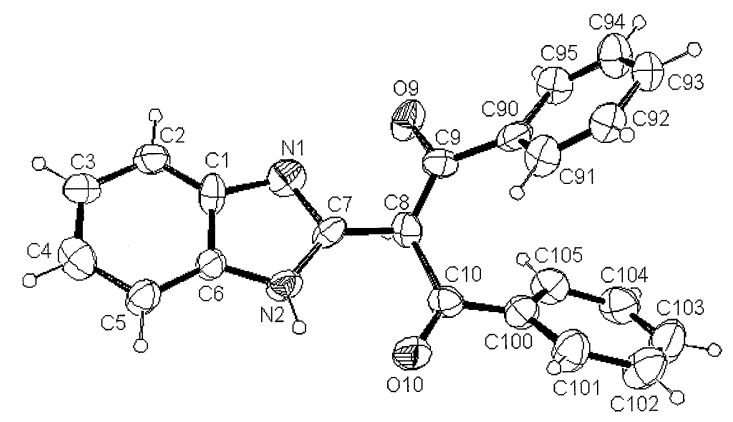

Fig. 2 ORTEP view of the title compound showing the labeling of the non-H atoms. Thermal ellipsoids are shown at the 30\% probability level.

\section{Acknowledgements}

The authors wish to acknowledge the purchase of the CAD-4 diffractometer under Grant DPT/TBAG1 of the Scientific and Technical Research Council of Turkey.

\section{References}

1. W. B. Pratt, "Chemotherapy of Infection", 1977, Oxford University Press, New York.

2. H. Göker and U. Abbasoğlu, Arch. Pharm. [Weinheim], 1995, 328, 425.

3. S. Özbey, E. Kendi, H. Göker, and M. Tunçbilek, J. Chem. Crsytallogr., 1998, 28, 461.

4. A. Aydın, H. Soylu, B. Güneş, M. Akkurt, F. Ercan, H. Küçükbay, and E. Çetinkaya, Z. Kristallogr., 1998, 213, 473.

5. Ş. K. İngeç, H. Soylu, H. Küçükbay, F. Ercan, and M. Akkurt, Anal. Sci., 1999, 15, 927.

6. Y. Elerman and M. Kabak, Acta Crystallogr., 1997, C53, 372.

7. S. Özbey, S. İde, and E. Kendi, J. Mol. Struct., 1998, 23, 442. 